\title{
Sobre a Dedução da Equação da Onda e da Solução Segundo a Fórmula de Kirchhoff
}

R.T. COUTO ${ }^{1}$, Departamento de Matemática Aplicada, Universidade Federal Fluminense (UFF), 24020-140 Campus do Valonguinho, Centro, Niterói, RJ, Brasil.

Resumo. Trata-se de novas deduções da equação da onda bem como da fórmula de Kirchhoff para a sua solução em meios sem fronteiras. Os métodos apresentados são suscetíveis de serem aplicáveis também no desenvolvimento de teorias nãoondulatórias.

\section{Introdução}

Este trabalho contribui de duas maneiras no estudo do fenômeno ondulatório, apresentando deduções originais da equação da onda e da solução segundo a fórmula de Kirchhoff.

Para expor o método de obtenção da equação da onda, consideramos um problema bidimensional: a membrana vibrante. A contribuição reside no fato de a dedução não se basear em um sistema de coordenadas particular. Isso contrasta com as deduções encontradas na literatura (e.g., referências [2] e [3]), que privilegiam as coordenadas cartesianas. Vantagens do método apresentado são a sua simplicidade e o insight proporcionado.

Quanto à fórmula de Kirchhoff, sabemos que ela fornece a solução da equação da onda em meios sem fronteiras (todo o $\mathbb{R}^{n}$ ). Uma demonstração construtiva da mesma, entretanto, é dificilmente encontrada na literatura. No $\mathbb{R}^{3}$, a prática rotineira consiste em enunciar a fórmula e então verificar que ela de fato fornece a solução (e.g., referências [8, Eq. (5,12)], [10, Sec. 9.2, Eq. (3)] e [12, p. 286, lema 5.3]). Já a fórmula válida no $\mathbb{R}^{2}$ é sempre obtida pelo método idealizado por Hadamard, descendo-se uma dimensão a partir do caso tridimensional (e.g., referências [4], [8, Eq. (13,12)], [10, Sec. 9.2, Eq. (19)] e [12, p. 289]). Neste trabalho, resolvendo a equação da onda em duas e três dimensões, deduzimos a fórmula de Kirchhoff.

A Seção 2 contém a dedução da equação da membrana. A Seção 3 apresenta a dedução da fórmula de Kirchhoff. A Seção 4 acresce comentários finais, encerrando a exposição.

\section{A Membrana Vibrante}

Considere uma membrana de forma arbitrária, que, quando estática, apresenta-se totalmente contida no plano $x y$ (horizontal) e esticada igualmente em todos os seus

\footnotetext{
${ }^{1}$ toscano@im.uff.br
} 
pontos e em todas as direções. Essa hipótese de esticamento uniforme e isotrópico pode ser entendida como segue:

A Figura 1 mostra essa membrana com um pequeno corte $\mathcal{A B}$ retilíneo e de comprimento $s$, não necessariamente infinitesimal. Mostramos as forças oriundas do esticamento que agem nos pontos ao longo de uma das margens do corte, as quais, por causa daquelas forças, tendem a se separar mais e mais. A uniformidade do esticamento implica que $F / s \equiv$ $T=$ constante em qualquer local da membrana onde se efetue um corte paralelo. Já a sua isotropia significa que, num mesmo local, a tensão por unidade de comprimento $T$ é constante em cortes de todas as direções. (Na ausência de uniformidade e isotropia, é necessário considerar um corte

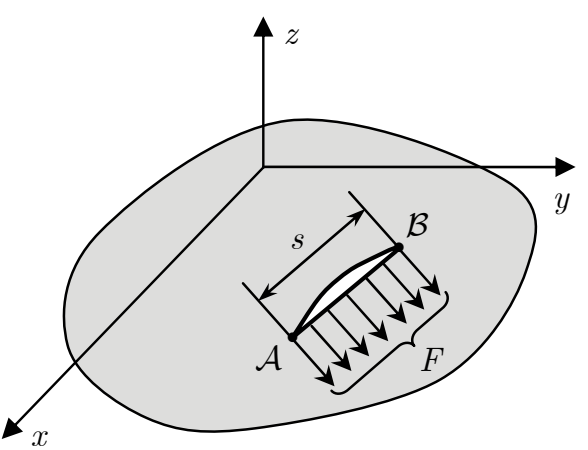

Figura 1: Forças numa das margens de um corte na membrana.

infinitesimal e o vetor $\overrightarrow{d s}$ ao longo do mesmo; nesse caso, $T$ depende do ponto $\vec{r}$ da membrana e da direção de $\overrightarrow{d s}$.) Observe que $T d s$ é a força com que a margem de um corte de comprimento $d s$ é puxada transversalmente.

Outras hipóteses são admitidas: (1) a forma da membrana pode ser descrita por uma função $z(x, y, t)$ que fornece, no instante $t$, a deflexão $z$, em relação ao plano $x y$, do ponto da membrana de abscissa $x$ e ordenada $y$; (2) ela nunca se inclina apreciavelmente (hipótese de pequenas inclinações): o módulo do ângulo de inclinação $|\theta|$ de qualquer reta tangente em qualquer dos pontos da membrana é suficientemente pequeno para que seja válida a aproximação $\cos \theta \simeq 1(\mathrm{e} \operatorname{sen} \theta \simeq$ $\tan \theta$, portanto); (3) as vibrações são verticais. Essas são hipóteses de restrição cinemática (uma limitação no movimento da membrana).

Admite-se também que a membrana: (1) seja homogênea, com densidade superficial $\rho=d m / d S$ constante; (2) só possa sofrer a atuação de forças externas que sejam verticais, sendo $\vec{f}(x, y, t)=f(x, y, t) \vec{e}_{z}$ a grandeza que fornece essas forças por unidade de área da membrana [por exemplo, $f=(-d m g) / d S=-\rho g$ se só a gravidade atua]. Essas são hipóteses de restrição dinâmica.

Preliminarmente, para maior clareza dos argumentos adotados na dedução a seguir, lembremo-nos da definição da derivada da função $z(x, y, t)=z(\vec{r}, t)$ na direção do vetor unitário $\vec{u}$ do plano $x y$ bem como a sua expressão que envolve o $\nabla z$, válida se $z$ for diferenciável (o que se admite):

$$
\frac{\partial z}{\partial \vec{u}}(\vec{r}, t) \equiv \lim _{h \rightarrow 0} \frac{z(\vec{r}+h \vec{u})-z(\vec{r}, t)}{h}=\vec{u} \cdot \nabla z(\vec{r}, t) .
$$

Em particular, se $\vec{u}$ for um dos versores cartesianos, a derivada direcional será nada mais do que uma derivada parcial: $\partial z / \partial \vec{e}_{x}=\partial z / \partial x$ e $\partial z / \partial \vec{e}_{y}=\partial z / \partial y$. Esse fato ajuda no entendimento de outro aspecto da derivada direcional. Sabemos que $[\partial z / \partial x]\left(x_{0}, y_{0}, t\right)$ é o coeficiente angular (a tangente do ângulo de inclinação) da 
reta tangente à curva de interseção do gráfico ${ }^{2}$ da função $z(x, y, t)$ com o plano que é paralelo ao plano coordenado $x z$ e passa pelo ponto $\left(x_{0}, y_{0}\right)$ do plano $x y$ (v. Figura $2 \mathrm{a}$, na qual $\tau$ é a reta tangente). Analogamente, $[\partial z / \partial \vec{u}]\left(\vec{r}_{0}, t\right)$ é o coeficiente angular da reta tangente à curva de interseção do mesmo gráfico com o plano vertical paralelo a $\vec{u}$ e passando pelo ponto $\vec{r}_{0}$ do plano $x y$ (v. Figura $2 \mathrm{~b}$ ).

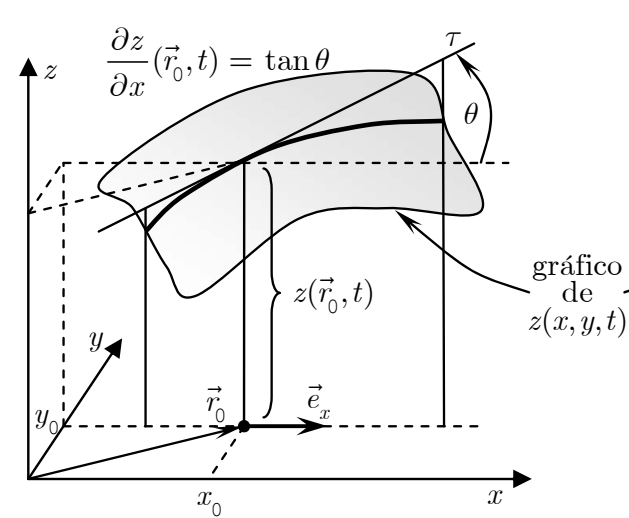

(a)

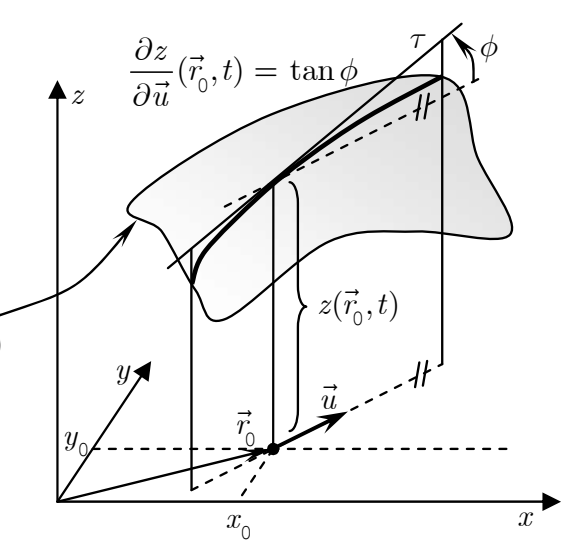

(b)

Figura 2: Interpretação geométrica da derivada da função $z(x, y, t)$, no ponto $\vec{r}_{0}=$ $\left(x_{0}, y_{0}\right)$ : (a) na direção do versor cartesiano $\vec{e}_{x}$; (b) na direção do vetor unitário $\vec{u}$.

Uma derivada direcional particular é a chamada derivada normal. Ela é a derivada ao longo do vetor unitário normal exterior $\vec{n}$, geralmente denotada na literatura por $\partial z / \partial n$ em vez de $\partial z / \partial \vec{n}$. Quando a função $z(\vec{r}, t)=z(x, y, t)$ descreve uma membrana, é comum calcular $\partial z / \partial n$ em pontos da curva $\mathcal{C}$ de projeção no plano $x y$ da borda $\Lambda$ da membrana (freqüentemente $\Lambda=\mathcal{C}$, i.e., a borda da membrana encontra-se fixa no plano $x y$ ). Nesse caso, os vetores unitários normais exteriores são vetores do plano $x y$ normais a $\mathcal{C}$, como os indicados na Figura 3, nos pontos $\vec{r}_{1}$ e $\vec{r}_{2}$. A derivada $[\partial z / \partial n]\left(\vec{r}_{1}, t\right)$ pode ser interpretada como o coeficiente angular de uma

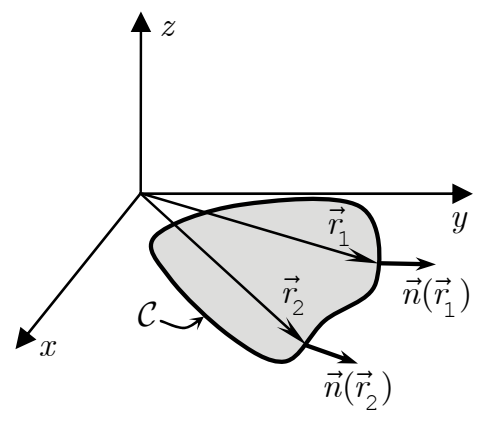

Figura 3: Vetores unitários normais à fronteira da região de projeção da membrana no plano $x y$.

ou como a taxa de variação de $z(\vec{r}, t)$, no ponto $\vec{r}=\vec{r}_{1}$ do domínio espacial dessa função, na direção de $\vec{n}\left(\vec{r}_{1}\right)$.

Bem, podemos agora, em poucos passos, deduzir a equação que descreve o movimento da membrana. Para isso, considere uma porção finita qualquer da membrana, $\Sigma$, cuja margem é a curva $\Gamma$ (como na Figura 4). Denotemos as projeções de $\Sigma$ e

\footnotetext{
${ }^{2}$ Tal gráfico, em que $t$ é mantido fixo, pode caracterizar a forma de uma membrana no espaço, no instante $t$.
} 


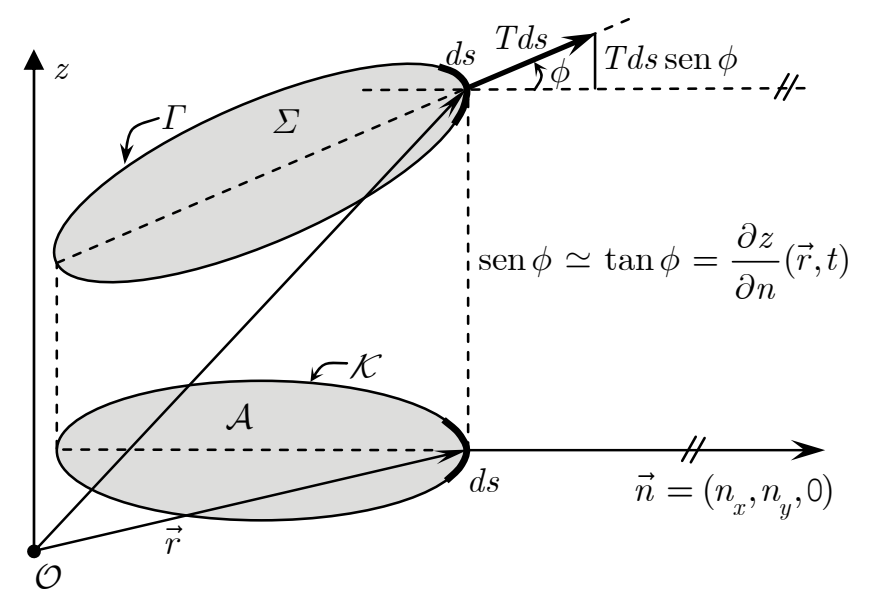

Figura 4: Uma porção $\Sigma$ da membrana e a força $T d s$ sobre o elemento de comprimento de arco $d s$ da sua margem $\Gamma$, bem como o vetor unitário normal $\vec{n}$ à fronteira $\mathcal{K}$ da projeção $\mathcal{A}$ de $\Sigma$ no plano $x y$.

de sua margem $\Gamma$ no plano $x y$ por $\mathcal{A}$ e $K$, respectivamente. Seja $\vec{n}=\left(n_{x}, n_{y}, 0\right)$ o vetor unitário normal exterior nessa fronteira $K$ de $\mathcal{A}$. Conforme já se mencionou, sobre um segmento $d s$ de $\Gamma$, atua uma tensão marginal de módulo $T d s$ que é direcionada para o exterior de $\Sigma$ e perpendicular à margem $\Gamma$. Isso, em conjunção com a hipótese de pequenas inclinações, permite concluir que o componente vertical dessa tensão é igual a $T d s[\partial z / \partial n]$ (v. Figura 4). Logo, aplicando em $\Sigma$ a 2 a lei de Newton na vertical, isto é, igualando o somatório das forças verticais sobre $\Sigma$ à massa $M$ dessa porção de membrana multiplicada pela aceleração do seu centro de massa, $z_{\mathrm{cm}}$, obtemos

$$
\int_{\Sigma} f d S+\oint_{\Gamma} T d s \operatorname{sen} \phi=M \frac{d^{2} z_{\mathrm{cm}}}{d t^{2}} \quad(\phi \text { como na Figura } 4) .
$$

Mas, pela hipótese de pequenas inclinações, temos que

$$
\int_{\Sigma} f d S \simeq \int_{\mathcal{A}} f d A
$$

e, conforme explicado no parágrafo anterior (e indicado na Figura 4), que

$$
\oint_{\Gamma} T d s \operatorname{sen} \phi \simeq \oint_{K} T d s \frac{\partial z}{\partial n}=T \oint_{K} d s \vec{n} \cdot \nabla z \stackrel{(\#)}{=} T \int_{\mathcal{A}} d A \nabla \cdot \nabla z=\int_{\mathcal{A}} T \nabla^{2} z d A,
$$

onde, na passagem (\#), usamos o teorema de Gauss no plano. Por outro lado, temos que

$$
M \frac{d^{2} z_{\mathrm{cm}}}{d t^{2}}=M \frac{d^{2}}{d t^{2}}\left(\frac{1}{M} \int_{\Sigma} z d m\right)=\frac{d^{2}}{d t^{2}}\left(\int_{\Sigma} z \rho d S\right) \simeq \frac{d^{2}}{d t^{2}} \int_{\mathcal{A}} z \rho d A=\int_{\mathcal{A}} \rho \frac{\partial^{2} z}{\partial t^{2}} d A .
$$


Logo, substituindo (2.2), (2.3) e (2.4) em (2.1), obtemos

$$
\int_{\mathcal{A}}\left(f+T \nabla^{2} z-\rho \frac{\partial^{2} z}{\partial t^{2}}\right) d A=0
$$

equação que, por ser a porção $\Sigma$ arbitrária (e também a sua projeção $\mathcal{A}$ ), só pode ser satisfeita se o integrando anular-se identicamente, o que nos leva à equação da membrana:

$$
\nabla^{2} z(\vec{r}, t)-\frac{1}{c^{2}} \frac{\partial^{2} z}{\partial t^{2}}=-\frac{1}{T} f(\vec{r}, t) \quad[c \equiv \sqrt{T / \rho}] .
$$

\section{A Fórmula de Kirchhoff}

Mostraremos que a solução da equação da onda

$$
\square^{2} v(\vec{r}, t) \equiv \nabla^{2} v-\frac{1}{c^{2}} \frac{\partial^{2} v}{\partial t^{2}}=0 \quad(t>0),
$$

em todo o plano ou todo o espaço (isto é, $\vec{r} \in \mathbb{R}^{n}$, com $n=2$ ou 3 ), sob as condições iniciais

$$
v(\vec{r}, 0)=0
$$

e

$$
\frac{\partial v}{\partial t}(\vec{r}, 0)=p(\vec{r})
$$

é dada pela fórmula de Kirchhoff:

$$
v(\vec{r}, t)=(2 \pi c)^{-1} \int_{\left|\vec{r}^{\prime}-\vec{r}\right|<c t} \frac{d A^{\prime} p\left(\vec{r}^{\prime}\right)}{\sqrt{c^{2} t^{2}-\left|\vec{r}^{\prime}-\vec{r}\right|^{2}}} \quad \text { se } n=2,
$$

em que a região de integração é a formada pelos pontos $\vec{r}^{\prime}$ tais que $\left|\vec{r}^{\prime}-\vec{r}\right|<c t$, ou seja, o disco de raio $c t$ e centro em $\vec{r}$, sendo $d A^{\prime}$ o elemento de área desse disco, ou

$$
v(\vec{r}, t)=\left(4 \pi c^{2} t\right)^{-1} \int_{\left|\vec{r}^{\prime}-\vec{r}^{\prime}\right|=c t} d S^{\prime} p\left(\vec{r}^{\prime}\right) \quad \text { se } n=3,
$$

em que a região de integração é a formada pelos pontos $\vec{r}^{\prime}$ tais que $\left|\vec{r}^{\prime}-\vec{r}\right|=c t$, ou seja, a superfície esférica de raio $c t$ e centro em $\vec{r}$, sendo $d S^{\prime}$ o elemento de área dessa superfície.

Convém fazer uma observação. É fácil verificar que a solução de $\square^{2} w(\vec{r}, t)=0$ sob as condições iniciais $w(\vec{r}, 0)=p(\vec{r})$ e $[\partial w / \partial t](\vec{r}, 0)=0\left(\operatorname{com} \vec{r} \in \mathbb{R}^{n}\right.$ e $\left.t>0\right)$ é dada por $w(\vec{r}, t)=[\partial v / \partial t](\vec{r}, t)$, onde $v(\vec{r}, t)$ é a solução do problema definido por (3.1), (3.2) e (3.3). Assim, a fórmula de Kirchhoff é suficiente para obter-se a solução da equação da onda em meios sem fronteiras mesmo sob condições iniciais que são ambas não-homogêneas, bastando recorrer-se ao princípio da superposição (cf. $[8$, Eq. $(4,12)]$ e [12, p. 286, lema 5.2]).

Para demonstrar a fórmula de Kirchhoff, dada por (3.4) ou (3.5) conforme o problema seja bi ou tridimensional, devemos resolver o problema de valor inicial 
formado pelas equações (3.1), (3.2) e (3.3), o que faremos por meio da transformada de Fourier

$$
\mathcal{F}\{v(\vec{r}, t)\}=\frac{1}{(2 \pi)^{n / 2}} \int_{\mathbb{R}^{n}} d^{n} r e^{i \vec{k} \cdot \vec{r}} v(\vec{r}, t) \equiv \bar{v}(\vec{k}, t) .
$$

Aplicando-a na equação (3.1) e resolvendo a EDO resultante, obtemos

$-k^{2} \bar{v}(\vec{k}, t)-c^{-2} \frac{d^{2} \bar{v}}{d t^{2}}=0 \Rightarrow \frac{d^{2} \bar{v}}{d t^{2}}+(k c)^{2} \bar{v}(\vec{k}, t)=0 \Rightarrow \bar{v}(\vec{k}, t)=C_{1} \cos k c t+C_{2} \operatorname{sen} k c t$.

Para determinar as constantes de integração $C_{1}$ e $C_{2}$, tomamos a transformada de Fourier das condições iniciais em (3.2) e (3.3), obtendo

$$
\bar{v}(\vec{k}, 0)=0 \quad \text { e } \quad \frac{d \bar{v}}{d t}(\vec{k}, 0)=\mathcal{F}\{p(\vec{r})\} \equiv \bar{p}(\vec{k}) .
$$

Exigindo que (3.7) satisfaça essas equações, determinamos $\bar{v}(\vec{k}, t)$ completamente:

$$
\left\{\begin{array}{l}
\bar{v}(\vec{k}, 0)=C_{1}=0 \\
\frac{d \bar{v}}{d t}(\vec{k}, 0)=k c C_{2}=\bar{p}(\vec{k}) \Rightarrow C_{2}=\frac{\bar{p}(\vec{k})}{k c} \quad \Rightarrow \quad \bar{v}(\vec{k}, t)=\frac{\bar{p}(\vec{k})}{c} \frac{\operatorname{sen} k c t}{k} .
\end{array}\right.
$$

A transformada de Fourier inversa desse resultado é a solução desejada, que pode ser calculada usando o teorema da convolução:

$v(\vec{r}, t)=\mathcal{F}^{-1}\left\{\frac{\bar{p}(\vec{k})}{c} \frac{\operatorname{sen} k c t}{k}\right\}=\frac{p(\vec{r})}{c} * G(\vec{r}, t)=\frac{1}{(2 \pi)^{n / 2} c} \int_{\mathbb{R}^{n}} d^{n} r^{\prime} p\left(\vec{r}^{\prime}\right) G\left(\vec{r}-\vec{r}^{\prime}, t\right)$,

onde

$$
\begin{gathered}
G(\vec{r}, t) \equiv \mathcal{F}^{-1}\left\{\frac{\operatorname{sen} k c t}{k}\right\}=\frac{1}{(2 \pi)^{n / 2}} \int_{\mathbb{R}^{n}} d^{n} k e^{-\mathrm{i} \vec{k} \cdot \vec{r}} \frac{\operatorname{sen} k c t}{k}= \\
\frac{1}{(2 \pi)^{n / 2}} \int_{\mathbb{R}^{n}} \frac{d^{n} k}{k} e^{-\mathrm{i} \vec{k} \cdot \vec{r}} \frac{e^{\mathrm{i} k c t}-e^{-\mathrm{i} k c t}}{2 \mathrm{i}}=\frac{I_{+}(\vec{r})-I_{-}(\vec{r})}{2 \mathrm{i}(2 \pi)^{n / 2}}
\end{gathered}
$$

com

$$
I_{ \pm}(\vec{r}) \equiv \int_{\mathbb{R}^{n}} \frac{e^{-\mathrm{i} \vec{k} \cdot \vec{r}} e^{ \pm \mathrm{i} k c t}}{k} d^{n} k
$$

Neste ponto, devemos prosseguir considerando separadamente os casos bi e tridimensional.

\subsection{Caso tridimensional $(n=3)$}

Para efetuar a integral em (3.11), tripla no espaço de $\vec{k}$, escrevemos o elemento de volume desse espaço em coordenadas esféricas, $d^{3} k=k^{2} \operatorname{sen} \theta d k d \theta d \varphi(\mathrm{em}$ vez das coordenadas cartesianas, $d^{3} k=d k_{x} d k_{y} d k_{z}$ ), com o cuidado de coincidir a direção do eixo $k_{z}$ com a do vetor $\vec{r}$, como mostra a Figura 5. Desse jeito, o ângulo entre $\vec{k}$ e $\vec{r}$ passa a ser a variável de integração $\theta$ e, portanto, $\vec{k} \cdot \vec{r}=k r \cos \theta$, o que simplifica consideravelmente as integrações em $\theta$ e $\phi$ : 


$$
\begin{gathered}
I_{ \pm}(\vec{r}) \equiv \int_{\mathbb{R}^{3}} \frac{e^{-\mathrm{i} \vec{k} \cdot \vec{r}} e^{ \pm \mathrm{i} k c t}}{k} d^{3} k \\
=\int_{0}^{2 \pi} \int_{0}^{\pi} \int_{0}^{\infty} \frac{e^{ \pm \mathrm{i} k c t} e^{-\mathrm{i} k r \cos \theta}}{k} k^{2} \operatorname{sen} \theta d k d \theta d \varphi \\
=\frac{2 \pi}{\mathrm{i} r} \int_{0}^{\infty} d k e^{ \pm \mathrm{i} k c t} \int_{0}^{\pi} d \theta e^{-\mathrm{i} k r \cos \theta} \mathrm{i} k r \operatorname{sen} \theta \\
=\frac{2 \pi}{\mathrm{i} r} \int_{0}^{\infty} d k e^{ \pm \mathrm{i} k c t}\left[e^{-\mathrm{i} k r \cos \theta}\right]_{\theta=0}^{\pi} \\
=\frac{2 \pi}{\mathrm{i} r} \int_{0}^{\infty} d k e^{ \pm \mathrm{i} k c t} \underbrace{\left[e^{\mathrm{i} k r}-e^{-\mathrm{i} k r}\right]}_{2 \mathrm{i} \operatorname{sen} k r} \\
=\frac{4 \pi}{r} \int_{0}^{\infty} d k e^{ \pm \mathrm{i} k c t} \operatorname{sen} k r .
\end{gathered}
$$

A substituição desse resultado em (3.10) fornece

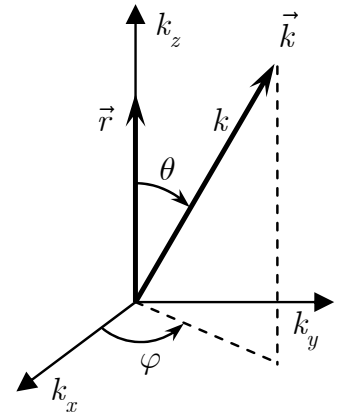

Figura 5: Os eixos das coordenadas cartesianas $\left(k_{x}, k_{y}, k_{z}\right) \mathrm{e}$ as coordenadas esféricas $(k, \theta, \varphi)$ do vetor $\vec{k}$.

$$
\begin{gathered}
G(\vec{r}, t)=\frac{1}{2 \mathrm{i}(2 \pi)^{3 / 2}} \frac{4 \pi}{r} \int_{0}^{\infty} d k \operatorname{sen} k r \underbrace{\left(e^{\mathrm{i} k c t}-e^{-\mathrm{i} k c t}\right)}_{2 \mathrm{i} \text { senkct }} \\
=\frac{4 \pi}{(2 \pi)^{3 / 2} r} \int_{0}^{\infty} d k \operatorname{sen} k r \operatorname{sen} k c t=\frac{4 \pi}{(2 \pi)^{3 / 2} r} \frac{\pi}{2} \delta(r-c t)=\frac{\sqrt{\pi / 2}}{|\vec{r}|} \delta(|\vec{r}|-c t),
\end{gathered}
$$

onde fizemos uso do seguinte lema (deduzido no Apêndice):

Lema 3.1.

$$
\frac{2}{\pi} \int_{0}^{\infty} d k \operatorname{senkx} \operatorname{senk} x^{\prime}=\delta\left(x-x^{\prime}\right) .
$$

Substituindo, por sua vez, (3.12) em (3.9), obtemos, finalmente,

$$
\begin{gathered}
v(\vec{r}, t)=\frac{1}{(2 \pi)^{3 / 2} c} \int_{\mathbb{R}^{3}} d^{3} r^{\prime} p\left(\vec{r}^{\prime}\right) \frac{\sqrt{\pi / 2}}{\left|\vec{r}-\vec{r}^{\prime}\right|} \delta\left(\left|\vec{r}-\vec{r}^{\prime}\right|-c t\right) \\
=\frac{1}{(2 \pi)^{3 / 2} c} \oint_{\left|\vec{r}-\vec{r}^{\prime}\right|=c t} d S^{\prime} p\left(\vec{r}^{\prime}\right) \frac{\sqrt{\pi / 2}}{c t}=\frac{1}{4 \pi c^{2} t} \oint_{\left|\vec{r}^{\prime}-\vec{r}\right|=c t} d S^{\prime} p\left(\vec{r}^{\prime}\right) .
\end{gathered}
$$

\subsection{Caso bidimensional $(n=2)$}

Nesse caso, convém expressar o elemento de área $d^{2} k=d k_{x} d k_{y}$ da integral dupla em (3.11) em coordenadas polares, isto é, $d^{2} k=k d k d \varphi$, bem como escolher o eixo $k_{x}$ na direção oposta à do vetor $\vec{r}$, como mostra a Figura 6 . Assim, notando que $\vec{k} \cdot \vec{r}=-r \cos \varphi$, podemos escrever 


$$
\begin{gathered}
I_{ \pm}(\vec{r})=\int_{\mathbb{R}^{2}} \frac{e^{-\mathrm{i} \vec{k} \cdot \vec{r}} e^{ \pm \mathrm{i} k c t}}{k} d^{2} k \\
=\int_{0}^{2 \pi} \int_{0}^{\infty} e^{ \pm \mathrm{i} k c t} e^{\mathrm{i} k r \cos \varphi} d k d \varphi .
\end{gathered}
$$

Não é evidente como prosseguir o cálculo dessa integral. Contrasta, agora, a ausência de $\operatorname{sen} \varphi$, que tornou óbvia a integração na variável $\varphi$ no caso tridimensional. Aqui, uma pista é reconhecer que

$$
\int_{0}^{2 \pi} e^{\mathrm{i} k r \cos \varphi} d \varphi=2 \pi \mathrm{J}_{0}(k r)
$$

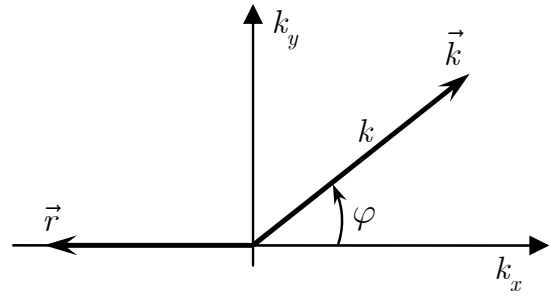

Figura 6: Os eixos das coordenadas cartesianas $\left(k_{x}, k_{y}\right)$ e as coordenadas polares $(k, \varphi)$ do vetor $\vec{k}$.

(v. a equação (11.30c) de [1]), o que permite escrever

$$
I_{ \pm}(\vec{r})=\int_{0}^{\infty} d k e^{ \pm \mathrm{i} k c t} \underbrace{\int_{0}^{2 \pi} d \varphi e^{\mathrm{i} k r \cos \varphi}}_{2 \pi \mathrm{J}_{0}(k r)}=2 \pi \int_{0}^{\infty} d k e^{ \pm \mathrm{i} k c t} \mathrm{~J}_{0}(k r) .
$$

Continuar a partir desse ponto é ainda menos evidente. Apresentamos o seguinte caminho, baseado na seguinte representação integral da função de Bessel em questão (v. a equação (14) do §6.21 de [11], na p. 180):

$$
\mathrm{J}_{0}(k r)=\frac{2}{\pi} \int_{1}^{\infty} \frac{\operatorname{sen} k r u}{\sqrt{u^{2}-1}} d u .
$$

Substituindo-a na equação anterior e invertendo a ordem das duas integrações, obtemos

$$
I_{ \pm}(\vec{r})=2 \pi \int_{0}^{\infty} d k e^{ \pm \mathrm{i} k c t} \frac{2}{\pi} \int_{1}^{\infty} d u \frac{\operatorname{sen} k r u}{\sqrt{u^{2}-1}}=4 \int_{1}^{\infty} \frac{d u}{\sqrt{u^{2}-1}} \int_{0}^{\infty} d k e^{ \pm \mathrm{i} k c t} \operatorname{sen} k r u .
$$

Agora substituímos esse resultado em (3.10) para escrever que

$$
\begin{aligned}
G(\vec{r}, t)= & \frac{4}{(2 \mathrm{i})(2 \pi)} \int_{1}^{\infty} \frac{d u}{\sqrt{u^{2}-1}} \int_{0}^{\infty} d k \underbrace{\left(e^{\mathrm{i} k c t}-e^{-\mathrm{i} k c t}\right)}_{2 \mathrm{i} \operatorname{sen} k c t} \operatorname{sen} k r u \\
& =\int_{1}^{\infty} \frac{d u}{\sqrt{u^{2}-1}} \underbrace{\left[\frac{2}{\pi} \int_{0}^{\infty} d k \operatorname{sen} k r u \operatorname{sen} k c t\right]}_{\delta(r u-c t)},
\end{aligned}
$$

onde já se encontra indicado que o termo entre colchetes, de acordo com (3.13), é uma representação integral da função delta abaixo dele. Mas $\delta(r u-c t)=r^{-1} \delta(u-$ $c t / r) ; \operatorname{logo}$,

$$
G(\vec{r}, t)=\frac{1}{r} \int_{1}^{\infty} \frac{d u \delta(u-c t / r)}{\sqrt{u^{2}-1}}=\frac{1}{r}\left\{\begin{array}{clll}
0 & \text { se } c t / r<1 \quad \text { i.e. } & c t-r<0 \\
\frac{1}{\sqrt{(c t / r)^{2}-1}} & \text { se } c t / r<1 \text { i.e. } c t-r>0
\end{array}\right.
$$




$$
=\frac{1}{r} \frac{1}{\sqrt{(c t / r)^{2}-1}} U(c t-r)=\frac{U(c t-|\vec{r}|)}{\sqrt{c^{2} t^{2}-|\vec{r}|^{2}}},
$$

onde $\mathcal{U}(x)$ denota a função degrau unitário, igual a 0 para $x$ negativo e a 1 para $x$ positivo.

Finalmente, a substituição do resultado acima em (3.9) produz a fórmula desejada

$$
v(\vec{r}, t)=\frac{1}{(2 \pi) c} \int_{\mathbb{R}^{2}} d^{2} r^{\prime} \frac{p\left(\vec{r}^{\prime}\right) U\left(c t-\left|\vec{r}^{\prime}-\vec{r}\right|\right)}{\sqrt{c^{2} t^{2}-\left|\vec{r}^{\prime}-\vec{r}\right|^{2}}}=(2 \pi c)^{-1} \int_{\left|\vec{r} \vec{r}^{\prime}\right|<c t} d^{2} r^{\prime} \frac{p\left(\vec{r}^{\prime}\right)}{\sqrt{c^{2} t^{2}-\left|\vec{r}^{\prime}-\vec{r}\right|^{2}}} .
$$

\section{Comentários Finais}

O método de dedução da equação da onda foi apresentado em duas dimensões e aplicado, em particular, ao problema da membrana vibrante; mas ele pode ser facilmente adaptado a outros problemas ondulatórios envolvendo qualquer número de dimensões espaciais.

A técnica concebida para levar a cabo a integração relacionada à dedução bidimensional da fórmula de Kirchhoff é susceptível de ser utilizada no cálculo de outras grandezas que envolvem uma integração similar (e.g., funções de Green).

Quanto às operações com a função delta como uma função - que não é! -, diga-se que ela simplifica consideravelmente os cálculos, pois, como se sabe, a função delta pode ser vista como um método abreviado de se obterem resultados que dependem de intrincados processos de limite. De fato, recordemos que Dirac não via no seu uso qualquer falta de rigor, antes afirmando ser sempre possível substituí-la por uma formulação equivalente, porém mais complicada (cf. [5]). Isso, de fato, confirmouse com a justificativa matemática da função delta que a teoria das distribuições propiciou, estando as suas principais propriedades rigorosamente estabelecidas na literatura, à nossa disposição, prontas para serem usadas (cf. [7] e [9]).

\section{Apêndice}

Deduzimos aqui o Lema 3.1. Ele pode ser obtido a partir das equações que definem a transformada de Fourier em senos,

$$
\mathcal{F}_{s}\{f(x)\}=\sqrt{\frac{2}{\pi}} \int_{0}^{\infty} d x^{\prime} f\left(x^{\prime}\right) \operatorname{sen} k x^{\prime} \equiv \bar{f}_{s}(k),
$$

e a transformada de Fourier em senos inversa,

$$
\mathcal{F}_{s}^{-1}\{f(x)\}=\sqrt{\frac{2}{\pi}} \int_{0}^{\infty} d k \bar{f}_{s}(k) \operatorname{sen} k x=f(x) .
$$

De fato, nessa segunda equação, substituindo $\bar{f}_{s}(k)$ por sua expressão dada pela primeira equação e invertendo a ordem das integrações, obtemos

$$
\int_{0}^{\infty} d x^{\prime} f\left(x^{\prime}\right)\left[\frac{2}{\pi} \int_{0}^{\infty} d k \operatorname{sen} k x \operatorname{sen} k x^{\prime}\right]=f(x)
$$


Ora, como $f(x)$ é uma função genérica, a equação acima é verdadeira se e somente se o termo entre colchetes é igual a $\delta\left(x-x^{\prime}\right)$.

Abstract. This works presents new derivations of the wave equation and of Kirchhoff's formula for its solution in media with no boundaries. The methods described are likely to be applicable in the development of other theories as well.

\section{Referências}

[1] G.B. Arfken, H.J. Weber, "Física Matemática", Campus, 6a ed., Rio de Janeiro, 2007.

[2] E. Butkov, "Física Matemática", LTC, Rio de Janeiro, 1988.

[3] R.V. Churchill, J.W. Brown, "Fourier Series and Boundary Value Problems", 3ㄹ ed., McGraw-Hill, 1978.

[4] R. Courant, D. Hilbert, "Methods of Mathematical Physics", vol. 2, WileyInterscience, 1962.

[5] P.A.M. Dirac, "The Principles of Quantum Mechanics", John Wiley \& Sons, 2 a ed., Nova Iorque, 1975.

[6] F. John, "Partial Differential Equations", 2a ed., Springer-Verlag, Nova Iorque, 1975.

[7] M.J. Lighthill, "Fourier Analysis and Generalised Functions", Cambridge U. P., Londres, 1964.

[8] I.G. Petrovsky, "Lectures on Partial Differential Equations", Interscience, 1954.

[9] L. Schwartz, "Théorie des distributions", Hermann, Paris, 1950.

[10] W.A. Strauss, "Partial Differential Equations, An Introduction", Wiley, 1992.

[11] G.N. Watson, "A Treatise on the Theory of Bessel Functions", 2a ed., Cambridge University Press, 1966.

[12] E.C. Zachmanoglou, D.W. Thoe, "Introduction to Partial Differential Equations with Applications", Dover, Nova Iorque, 1986. 